\title{
Medular atrophy progression in Friedreich Ataxia
}

\author{
Pedro Cury Moyses*, Marcondes Cavalcante França Jr
}

\begin{abstract}
Friedreich's Ataxia (FRDA) is the most common recessive autosomal ataxia, with early, poor prognosis and few treatment options. Despite established clinical scales are available for the follow-up of patients funcionality, little is known about the actual nervous system damage progression. In this article, we intended to analyze the progression of spinal cord injury over time in comparison with clinical deterioration progression and also with spinal cord injury progression in normal individuals. The analysis could have enabled using spinal cord damage measures over time as a biomarker for progression of FRDA, making posible to use it in clinical trials as na objetive to stop or delay the disease's progression.
\end{abstract}

\section{Key words:}

Friedreich, Medullar, Degeneration.

\section{Introduction}

Friedreich's Ataxia (FRDA) is the most common recessive autosomal ataxia, with early, poor prognosis and few treatment options. Despite established clinical scales are available for the follow-up of patients funcionality, little is known about the actual nervous system damage progression.

The cervical cord seems to be the first area affected by degeneration, followed by other neurological areas. It can even cause injury to other tissues and organs of the body, leading to cardiomyopathy, scoliosis and diabetes, for example. The systemic action is explained by its pathophysiology, related to the depletion of the frataxin protein, the result of the anomal reproduction of the GAA trinucleotide in the first intron of the FXN gene (chromosome 9q13)1.

The relationship between cervical spinal cord injury and the clinical status of an individual has been demonstrated in previous cross-sectional studies. The literature lacks, however, longitudinal research on the progression of cervical spinal cord neurodegeneration.

In this article, we intended to analyze the progression of spinal cord injury over time in comparison with clinical deterioration progression and also with spinal cord injury progression in normal individuals. The analysis could have enabled using spinal cord damage measures over time as a biomarker for progression of FRDA, making posible to use it in clinical trials as an objetive to stop or delay the disease's progression.

\section{Results and Discussion}

To do so, we used SpineSeg² software, developed by the Neuroimaging Laboratory of the State University of Campinas (Unicamp), an instrument already proven powerful in similar research, such as the cross-sectional FRDA study discussed above.

In the structural elaboration of this article, patients with FRDA (subjects) and control cases were separated, matched in gender and age, it was acquired magnetic resonance images of all individuals and the clinical scales of the subjects. Through the SpineSeg, the estimated measurement of the area and eccentricity of the cervical spinal cord in at least two distinct periods were collected in a standardized manner with the least possible bias.

After the data were collected, the patient groups and controls were organized. The FARS (Friedreich Ataxia
Rating Scale) clinical scale was attributed to the patients at each date of acquisition of the analyzed images. Three groups were then drawn, each with the same number of patients and controls, matched by gender and age, with the only difference being the interval between the date of the images. The first group consisted of 22 patients ( 9 men, 13 women) and 22 controls (9 men, 13 women), with intervals between 0.6 and 1.5 years. The second group had 9 patients (4 men, 5 women) and 9 controls ( 4 men, 5 women), ranging from 2 to 2.7 years. The third group had 4 patients (2 men, 2 women) and 4 controls ( 2 men, 2 women), ranging from 5 to 5.7 years.

We used descriptive statistics to expose the sample data. We used the ANOVA test for repeated measurements, with covariation for age and sex. The correlation between worsening of atrophy and functional decline will be yet explored with linear regression.

All analyzes were done in SYSTAT v.12 software. Values of $p<0.05$ would be considered significant.

On the three groups, there was no significant difference between the progression of spinal cord degeneration in patients and control along time, despite the cross-sectional differences. Therefore, we failed on stablishing a biomarker based on neuroimaging for FRDA damage progression.

\section{Conclusions}

We concluded that the main reason for failure was due to the slow progression of neurological damage in this ataxia. Therefore, since the first group (of 22 patients) did not show significance and the group images interval was at most 1.5 years, we assumed that greater evidence of spinal cord injury may be perceptible in the longitudinal analysis that contemplates longer time between images, as 2.5 and 5 years (groups of which we disposed of small amountof individuals).

\footnotetext{
1 Campuzano, V.; Montermini, L.; Molto et al. Friedreich's ataxia: autosomal recessive disease caused by an intronic GAA triplet repeat expansion. Science. 1996, 271:1423-1427.

${ }^{2}$ Bergo, F.; França, M.C.JR.; Chevis, C.F.; Cendes,F. SpineSeg: A segmentation and measurement tool for evaluation of spinal cord atrophy. Proceeding of the 7th Iberian Conference on Information Systems and Technologies (CISTI). 2012, 2. 400-403.
} 